\title{
Sensory-Specific Associations Stored in the Lateral Amygdala Allow for Selective Alteration of Fear Memories
}

\author{
Lorenzo Díaz-Mataix, ${ }^{1}$ Jacek Dębiec, ${ }^{1,2,3}$ Joseph E. LeDoux, ${ }^{1,4}$ and Valérie Doyère ${ }^{1,5,6}$ \\ ${ }^{1}$ Center for Neural Science, New York University, New York, New York 10003, ${ }^{2}$ New York University Child Study Center, New York, New York 10016, \\ ${ }^{3}$ Copernicus Center for Interdisciplinary Studies, Krakow 30387, Poland, ${ }^{4}$ Emotional Brain Institute, Nathan S. Kline Institute for Psychiatry Research, \\ Orangeburg, New York 10962, 5Université Paris-Sud, Centre de Neurosciences Paris-Sud, UMR 8195, Orsay F-91405, France, and ${ }^{6}$ CNRS, Orsay F-91405, \\ France
}

Consolidated long-term fear memories become labile and can be disrupted after being reactivated by the presentation of the unconditioned stimulus (US). Whether this is due to an alteration of the conditioned stimulus (CS) representation in the lateral amygdala (LA) is not known. Here, we show in rats that fear memory reactivation through presentation of the aversive US, like CS presentation, triggers a process which, when disrupted, results in a selective depotentiation of CS-evoked neural responses in the LA in correlation with a selective suppression of CS-elicited fear memory. Thus, an aversive US triggers the reconsolidation of its associated predictor representation in LA. This new finding suggests that sensory-specific associations are stored in the lateral amygdala, allowing for their selective alteration by either element of the association.

\section{Introduction}

In Pavlovian auditory fear conditioning, an initially neutral conditioned acoustic stimulus (CS), after being associated with an aversive unconditioned stimulus (US), typically electric shock, acquires the ability to induce conditioned fear responses (Pavlov, 1927). This form of associative learning critically involves the lateral nucleus of the amygdala (LA). Although the LA is believed to be a storage site for such fear memories (Davis, 1997; Fanselow and LeDoux, 1999; LeDoux, 2000; Maren, 2001; Fanselow and Poulos, 2005), the nature of the information stored in LA is unclear. A key issue is whether CS-US associations encoding specific sensory features of both stimuli are stored in the LA.

After consolidation of the fear memory, presentation of the US triggers a reconsolidation process which, when disrupted, leads to a decrease in CS-evoked behavioral fear memory in an amygdala-dependent manner (Debiec et al., 2010). It has been proposed that the neural representation of the CS activates sensory-specific representations of the US, which, in turn, elicits the corresponding behavioral conditioned responses (Konorski, 1967). Thus, the loss of fear behavior when US-triggered reconsolidation is disrupted may conceivably result from the disruption of

Received Nov. 4, 2010; revised April 16, 2011; accepted May 9, 2011.

Author contributions: L.D.-M., J.D., and V.D. designed research; L.D.-M. and V.D. performed research; L.D.-M. and V.D. analyzed data; L.D.-M., J.E.L., and V.D. wrote the paper.

This work was supported by the National Institute for Mental Health Grants R01 MH46516 and R01 MH38774, and McDonnell Foundation Grant 220020155. L.D.-M. was recipient of a Fulbright-Spanish Ministry of Science postdoctoral fellowship. We thank Dr. Joshua Johansen for his helpful suggestions revising the manuscript and Claudia Farb for her excellent assistance with histology.

The authors declare no competing financial interests.

This article is freely available online through the J Neurosci Open Choice option.

Correspondence should be addressed to Lorenzo Díaz-Mataix, Center for Neural Science, New York University, 4 Washington Place, Room 809, New York, NY 10003. E-mail: Idm5@nyu.edu.

DOI:10.1523/JNEUROSCI.5808-10.2011

Copyright $\odot 2011$ the authors $\quad 0270-6474 / 11 / 319538-06 \$ 15.00 / 0$ either CS or US neural representation. Using a second-order conditioning paradigm, we have previously shown that not all the fear associations are rendered labile in an amygdaladependent manner after reactivation of one of them (Debiec et al., 2006). It is therefore possible, as one could argue, that although amygdala-dependent, the behavioral disruption may reflect alteration of processes in brain networks other than the LA. Furthermore, even if some altered processes reside in the LA, they may not necessarily affect the CS representation, as a disrupted US representation may solely be responsible for the loss of behavioral-conditioned responses after disruption of US-triggered reconsolidation processes. Alternatively, stored first-order CS-US association in the LA could permit the CS representation to be rendered labile after US reactivation and produce a sensory-specific fear memory loss. A selective updating of CS representation would provide evidence that specific CS-US associations linked through neural plasticity are stored in the LA.

In the present study, we thus asked whether US reactivation triggers an update of the CS fear representation in the LA and produces a reconsolidation process during which the CS representation is destabilized. To do this, we recorded CS-evoked field potentials in LA and tracked the effects of US-induced reconsolidation blockade on the CS representation in the LA. We used an MAPK inhibitor to block the reconsolidation process, allowing the comparison of our result with previous data showing that the MAPK/ERK pathway is involved in reconsolidation triggered by the CS (Duvarci et al., 2005; Doyère et al., 2007). To control for the selectivity of the effects, we used a dual-fear conditioning protocol in which rats were trained to two different conditioned stimuli paired with two different aversive unconditioned stimuli (Debiec et al., 2010). We found that the blockade of US-triggered reconsolidation results in a selective erasure of the CS neural representation previously linked to that US but not of the CS linked to the other US. 
A

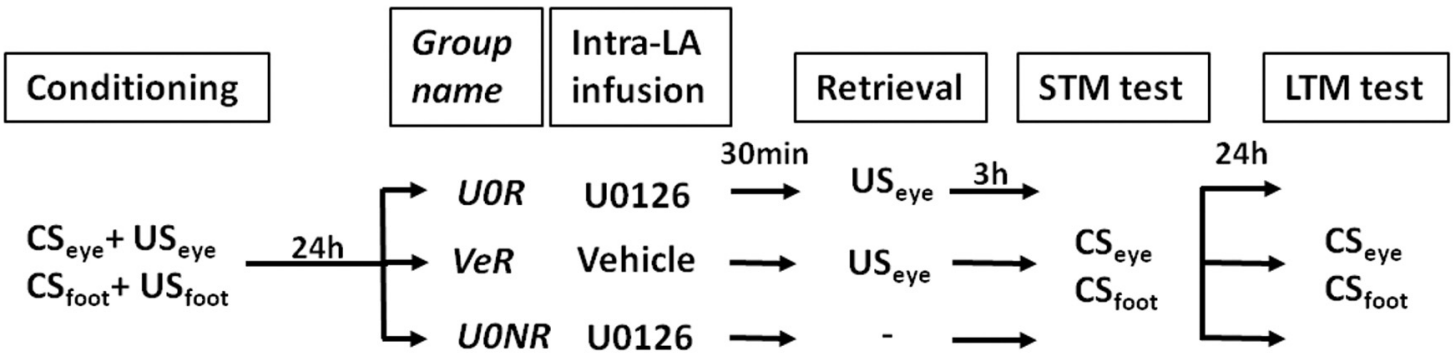

B
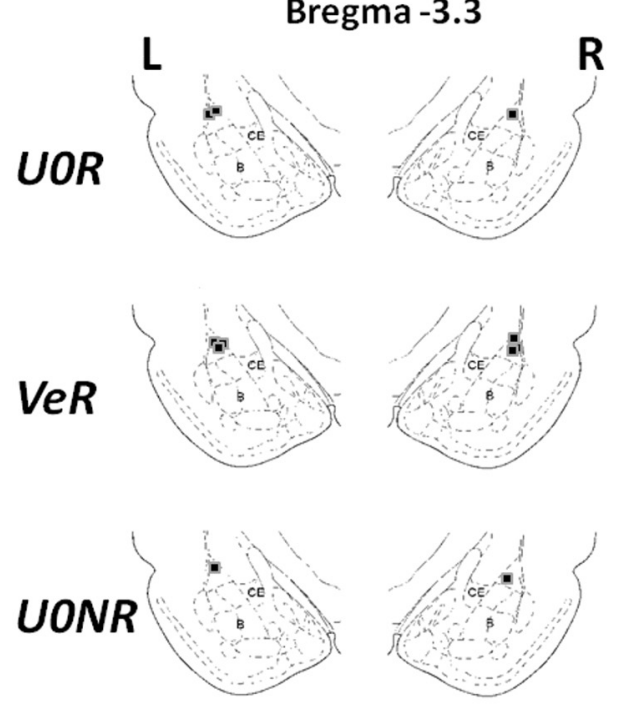

C
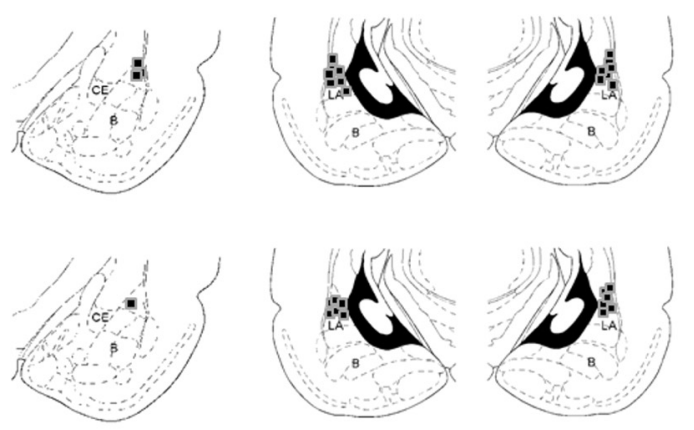

Bregma -3.8
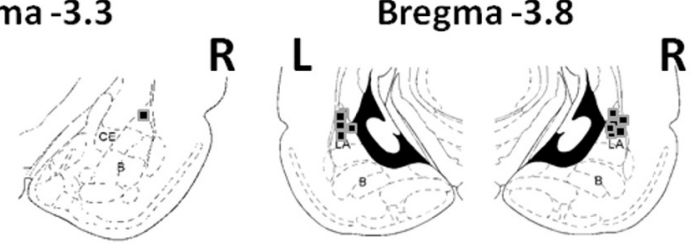

$\mathbf{R}$
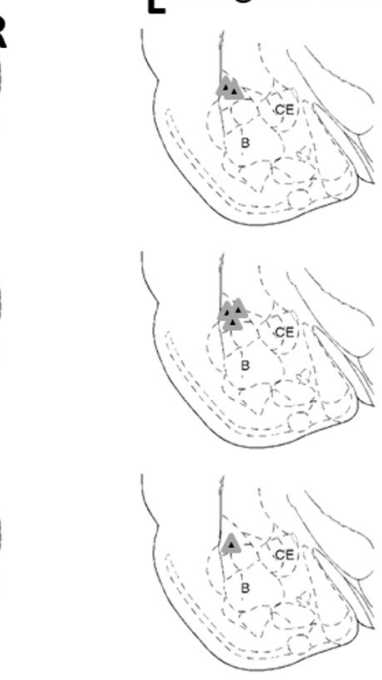

Bregma-3.3
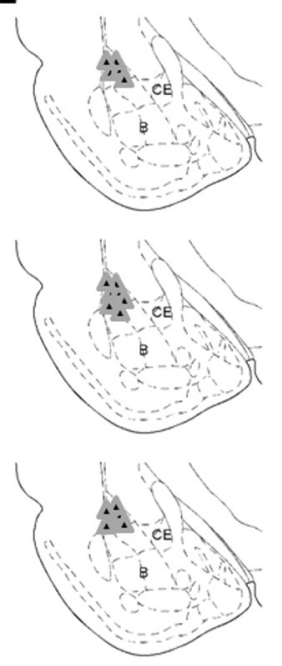

Figure 1. Experimental protocol and anatomical placement of cannulae and electrodes. A, Schematic of the main experimental design. After animals were trained in a two-CS and two US fear-conditioning paradigm, $\mathrm{CS}_{\text {eye }}$ paired with $\mathrm{US}_{\text {eye }}$ and $\mathrm{CS}_{\text {foot }}$ paired with $\mathrm{US}_{\text {foot }}$ rats were split into three different groups. The next day, 30 min before reactivation, two of the groups received the MAPK inhibitor U0126 (UOR, $n=7$; and UONR, $n=7$ ) and the third received vehicle (VeR, $n=10)$. One of the groups that received the MAPK inhibitor was not reactivated (UONR), while the other

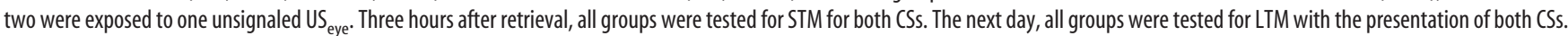
$\boldsymbol{B}, \boldsymbol{C}$, Anatomical placements of cannulae $(\boldsymbol{B})$ and electrodes $(\boldsymbol{C})$. The numbers represent the posterior coordinate from bregma for each schematic section of the amygdala. L, Left hemisphere; $R$, right hemisphere; $L A$, lateral nucleus of the amygdala; $B$, basal nucleus of the amygdala; $C E$, central nucleus of the amygdala.

\section{Materials and Methods}

Animals. Subjects were male Sprague Dawley rats (Hiltop Laboratories, Scottdale, PA) weighing 250-300 g at the beginning of the procedures. Rats were housed individually in plastic Nalgene cages and maintained on a 12/12 h light/dark cycle. Food and water were provided ad libitum. All procedures were in accordance with the National Institutes of Health Guide for the Care and Use of Experimental Animals and were approved by the New York University Animal Care and Use Committee.

Surgery. Rats were anesthetized with Nembutal (45 mg/kg, i.p.) and implanted bilaterally with cannulas (26 gauge; Plastics One, Roanoke, $\mathrm{VA}$ ) aimed at the LA (anteroposterior [AP], -3.2; mediolateral [ML], \pm 5.2 ; dorsoventral [DV], -8.0$)$ and a bipolar stimulation electrode (250 $\mu \mathrm{m}$; Rhodes Medical, Summerland, CA) aimed at the left auditory thalamus (medial geniculate nucleus/posterior intralaminar nucleus [MGm/ $\mathrm{PIN}]$; AP, -5.4 ; ML, $\pm 3.0 ; \mathrm{DV}, \sim 5.7$ from the brain surface). Attached to the left cannula was an insulated stainless steel recording wire (1-2 $\mathrm{M} \Omega$ ) (FHC, Inc.) that extended $0.5 \mathrm{~mm}$ from the base of the infusion cannula. The final depth of the recording electrode was determined using stimulation of the MGm/PIN as a guide to maximize the LA field potential amplitude (Doyère et al., 2007). Two silver balls, placed contralaterally above the neocortex, served as a reference and ground. For delivery of the periorbital shock, US two stainless steel wires (75 $\mu \mathrm{m}$ diameter, stripped of insulation $2 \mathrm{~mm}$ from the tip) were threaded through the skin of each eyelid (Debiec et al., 2010). All components were affixed to the skull using dental cement and acrylic, bound to three anchoring screws.
Rats were administered with ketoprofen $(2 \mathrm{mg} / \mathrm{kg}$ ) for analgesia and given at least 1 week to recover before the initiation of the behavioral procedures.

Electrophysiological and behavioral apparatus and stimuli. The apparatus and the tones used as two conditioned stimuli "eye" and "foot" $\left(\mathrm{CS}_{\text {eye }}, \mathrm{CS}_{\text {foot }}\right)$ were described in earlier studies (Doyère et al., 2007; Debiec et al., 2010). In brief, $\mathrm{CS}_{\text {eye }}$ was a $20 \mathrm{~s}$ series of acoustic pips $(1 \mathrm{kHz}, 50 \mathrm{~ms}$ duration, $1 \mathrm{~ms}$ ramp, $20 \mathrm{~dB}$ higher than the background noise, delivered at $1 \mathrm{~Hz}$ ), and $\mathrm{CS}_{\text {foot }}$ was a $20 \mathrm{~s}$ series of frequency modulation sweeps $(12.5 \mathrm{kHz}$ carrier frequency, $50 \mathrm{~Hz}$ modulation frequency, $2.5 \mathrm{kHz}$ modulation depth, $250 \mathrm{~ms}$ duration). The $\mathrm{US}_{\text {foot }}$ was a $0.5 \mathrm{~s}, 1.5 \mathrm{~mA}$ electric foot shock delivered through the grid floor (Model E10-10, Coulbourn Instruments). The $\mathrm{US}_{\text {eye }}$ was a $1.2 \mathrm{~s}$ train of five very brief shock pulses ( $3 \mathrm{~mA}$ for $2 \mathrm{~ms}$ ) delivered at a rate of $4 \mathrm{~Hz}$ to both eyes simultaneously. The US began immediately after the offset of the final pip.

Stimulus presentation was automated using Spike2 software (CED, Cambridge UK). Electrical signal was enhanced and filtered (gain, X10000; low pass, $0.1 \mathrm{~Hz}$; high pass, $1000 \mathrm{~Hz}$ ) using a model 1700 differential AC amplifier (A-M Systems) and transformed into a digital signal through a power 1401 CED interface.

Behavioral procedures. Here, we used a modification of a dual-fear conditioning paradigm used in our previous paper (Debiec et al., 2010).

Habituation to context (days 1 and 2). Rats were placed in the conditioning chamber (context A) and habituated to connecting cables for 30 min on 2 consecutive days. 
For all stages of the experiments, the session started with an acclimation period of $120 \mathrm{~s}$, and the intertrial interval was variable (130 s on average).

CS pre-exposure (days 3 and 4). Rats received three pre-exposures of one of the CS followed by three pre-exposures of the other CS in context $\mathrm{A}$, and the order was reversed on each day.

Conditioning (day 5). In context A, three CSeye $-\mathrm{US}_{\text {eye }}$ and three $\mathrm{CS}_{\text {foot }}-\mathrm{US}_{\text {foot }}$ pairings were given. The US was delivered immediately after the end of each CS.

Memory reactivation (day 6 ). In context $\mathrm{A}$, a single $\mathrm{US}_{\text {eye }}$ was presented with the same intensity and duration than the conditioning $\mathrm{US}_{\text {eye }}$. Thirty minutes before reactivation, rats were infused bilaterally with 1,4-diamino-2,3-dicyano1,4-bis(2-aminophenylthio)-butadiene (U0126) (1 $\mu \mathrm{g} ; 0.5 \mu \mathrm{l}$ in $50 \%$ DMSO) (U0R group) or an equivalent volume of vehicle ( $50 \%$ DMSO) (VeR group). This dose of U0126 was chosen based on findings in previous studies showing that it impairs fear memory reconsolidation (Duvarci et al., 2005, Doyère et al., 2007). The third group of rats (U0NR) were infused with the U0126 but were not exposed to the $\mathrm{US}_{\text {eye }}$ shock nor to the context.

Short-term memory retention tests. Three hours after the reactivation trial, rats were placed in a modified context with a peppermint-scented black floor (context B) and tested for short-term memory retention (STM). Four presentations of $\mathrm{CS}_{\text {eye }}$ were presented, followed $180 \mathrm{~s}$ later by four presentations of $\mathrm{CS}_{\text {foot }}$.

Long-term memory retention tests (day 7). Approximately $24 \mathrm{~h}$ after reactivation, rats were given a long-term memory retention (LTM) test. Nine $\mathrm{CS}_{\text {eye }}$ and nine $\mathrm{CS}_{\text {foot }}$ were presented in context $\mathrm{B}$ following the same order as in the STM test $\left(\mathrm{CS}_{\text {eye }}\right.$, followed by $\left.\mathrm{CS}_{\text {foot }}\right)$.

Reinstatement procedure (day 8). The day after LTM test, the group of rats with impaired fear memory (U0R group) was placed in the chamber with the original context (context A), and an unsignaled $\mathrm{US}_{\text {eye }}$ of the same duration and intensity as in conditioning was delivered to reinstate the lost memory.

Postreinstatement long-term memory test (day 9). Twenty-four hours after reinstatement, rats were placed in the context $\mathrm{B}$ for postreinstatement longterm memory (LTMpR). Four presentations of $\mathrm{CS}_{\text {eye }}$ were presented.

Reinstatement protocol after extinction. A different group of rats were implanted in each eyelid with two stainless steel wires ( $75 \mu \mathrm{m}$ diameter, stripped of insulation $2 \mathrm{~mm}$ from the tip) for delivery of the periorbital shock US $\left(\mathrm{US}_{\text {eye }}\right.$ ). After $7 \mathrm{~d}$ of recovery from the surgery, rats were placed in the conditioning chamber (context $\mathrm{A}$ ) and were fear-trained in the $\mathrm{CS}_{\text {eye }}-\mathrm{US}_{\text {eye }}$ and $\mathrm{CS}_{\text {foot }}-\mathrm{US}_{\text {foot }}$ protocol. Twenty-four hours after conditioning, rats were placed in the chamber (context A) and were submitted to 2 consecutive days of strong extinction with the presentation of 15 $\mathrm{CS}_{\text {eye }}$ and $15 \mathrm{CS}_{\text {foot }}$ each day; the order was reversed on each day. The day after the last extinction session, one unsignaled $\mathrm{US}_{\text {eye }}$ (same duration and intensity as in conditioning) was delivered to all the rats in context A. Twenty-four hours later, all rats were tested for LTMpR with four presentations of the $\mathrm{CS}_{\text {eye }}$ followed by four presentations of the $\mathrm{CS}_{\text {foot }}$ in a modified context with peppermint floor (context B).

Recordings. Rats' freezing behavior was recorded onto videotape during all sessions for offline blind scoring. During pre-exposure and retention tests, auditory-evoked field potentials (AEFPs) were recorded from LA, and blindly analyzed using Spike2 software. AEFPs were averaged across all tone-pip presentations within a trial and collapsed across trials. The amplitude of the short latency negative component was measured for each potential $(\sim 12-16 \mathrm{~ms})$. Time of freezing (s) and amplitude of AEFP $(\mu \mathrm{V})$ were averaged from four CS presentations within every session.
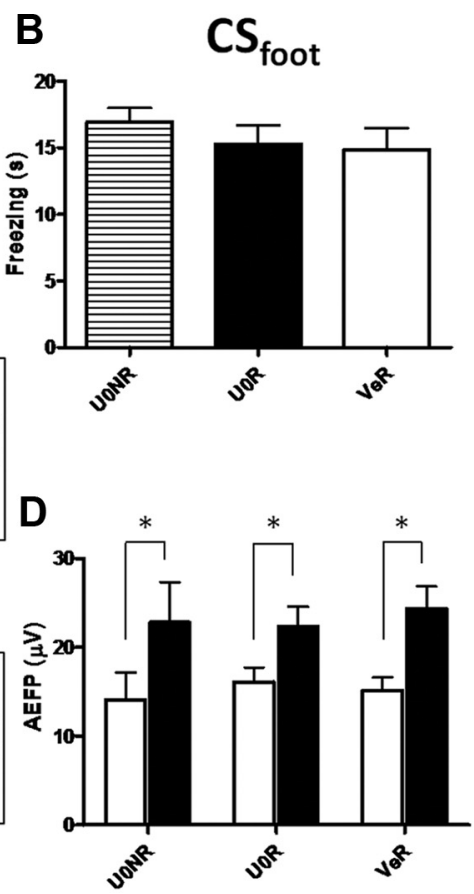

Figure 2. Fear conditioning with a two CSs and USs training protocol induces potentiation of both CS-evoked neurophysiologexamples of AEFPs evoked by pure tone $\left(\mathrm{CS}_{\text {eye }}\right)($ top $)$ and frequency-modulated tone $\left(\mathrm{CS}_{\text {foot }}\right)$ (down) recorded in lateral amygdala (STM). Calibration: $10 \mu \mathrm{V}, 5 \mathrm{~ms}$.

Histology. To check the brain placement of the cannulae and electrodes, at the end of the experiment, rats were anesthetized with an overdose of chloral hydrate $(600 \mathrm{mg} / \mathrm{kg}$, i.p.), and the location of the electrodes was marked by passing a small amount of anodal current $(4 \mu \mathrm{A} ; 5 \mathrm{~s})$ through the electrode tips. Rats were then perfused with $10 \%$ buffered formalin, and brains were sectioned and stained with Nissl for analysis of electrode and cannulae placement. Only the animals with both cannulae and electrode placed in the lateral amygdala were included in the analysis.

Statistical analysis. Data were analyzed by using one-way ANOVA (for the freezing analysis) or two-way ANOVA (for the AEFP analysis) with group (U0R, U0NR, VeR) as between-subject factors and session (habituation $[\mathrm{HAB}]$, STM, LTM) as within-subject factor. Significant effects were analyzed using a single interaction and a post hoc Tukey's honest significant difference test where appropriate.

\section{Results}

No contextual freezing was evident in any reactivation or test phases of the experiments (Fig. 1) (maximum group mean of preCS levels of freezing of $4.2 \mathrm{~s}$ ). The preCS levels of freezing were not statistically different between the different experimental groups ( $F$ s $>1$; data not shown).

Does the US-triggered reactivation of fear memory induce a change in the associated CS representation in LA?

During the STM test, all groups expressed equivalent freezing to $\mathrm{CS}_{\text {eye }}$ as well as to $\mathrm{CS}_{\text {foot }}$ (no group main effects: $F \mathrm{~s}<1$ in both cases) (Fig. 2A,B), showing that neither the MAPK inhibitor, nor the $\mathrm{US}_{\text {eye }}$ reactivation, affected short-term fear memory after US reactivation. As previously reported, fear conditioning led to a potentiation in the LA (Rogan et al., 1997; Schafe et al., 2005; Doyère et al., 2007). This was observed for both $\mathrm{CS}_{\text {eye }}$ and $\mathrm{CS}_{\text {foot }}$ as an increase in the amplitude of the AEFP during STM com- 

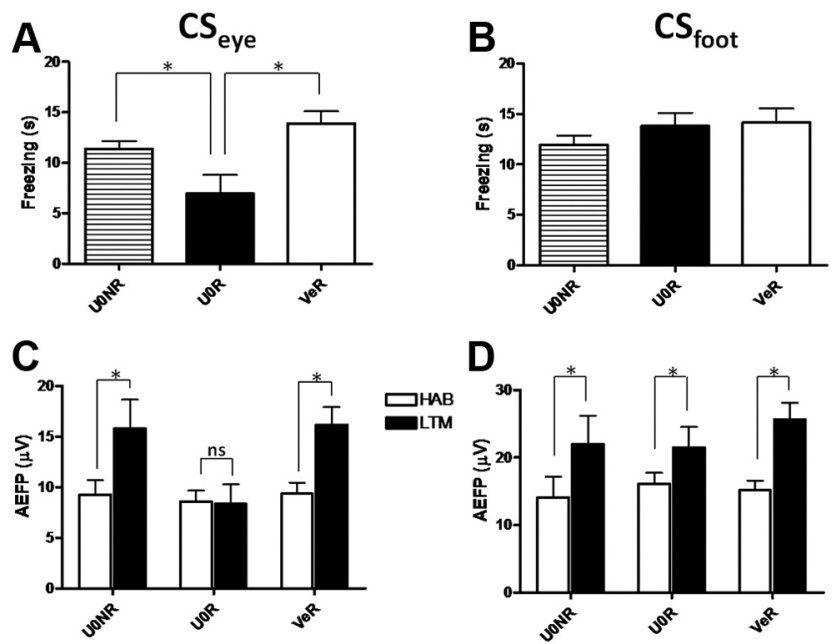

Figure 3. The selective impairment of memory due to the blockade of US-triggered reconsolidation correlates with a selective depotentiation of the $C S$-evoked field potential in lateral amygdala. $\boldsymbol{A}, \boldsymbol{B}$, Freezing duration (mean $\pm \mathrm{SEM}$ ) was scored for $\mathrm{CS}_{\text {eye }}(\boldsymbol{A})$ or $\mathrm{CS}_{\text {foot }}(\boldsymbol{B})$ presentations during LTM tests. Only the UOR group showed a loss of freezing selectively to the $C_{\text {eye }}$ C,$D$, Amplitude of the short-latency AEFP in LA (mean \pm SEM) evoked by the $C_{\text {eye }}(\boldsymbol{C})$ and by the $C S_{\text {foot }}(\boldsymbol{D})$ is represented for HAB (white bars) and LTM test (black bars). Going in parallel with the behavior, only the UOR group showed a depotentiation of the $\mathrm{CS}_{\text {eye }}-\mathrm{AEFP}$, while maintaining the potentiation of the $\mathrm{CS}_{\text {foot }}-\mathrm{AEFP}$. AEFPs of the two control groups (VeR and UONR) remained potentiated to both CSs $\left({ }^{*} p<0.05\right.$, Tukey post hoc test $)$.

A
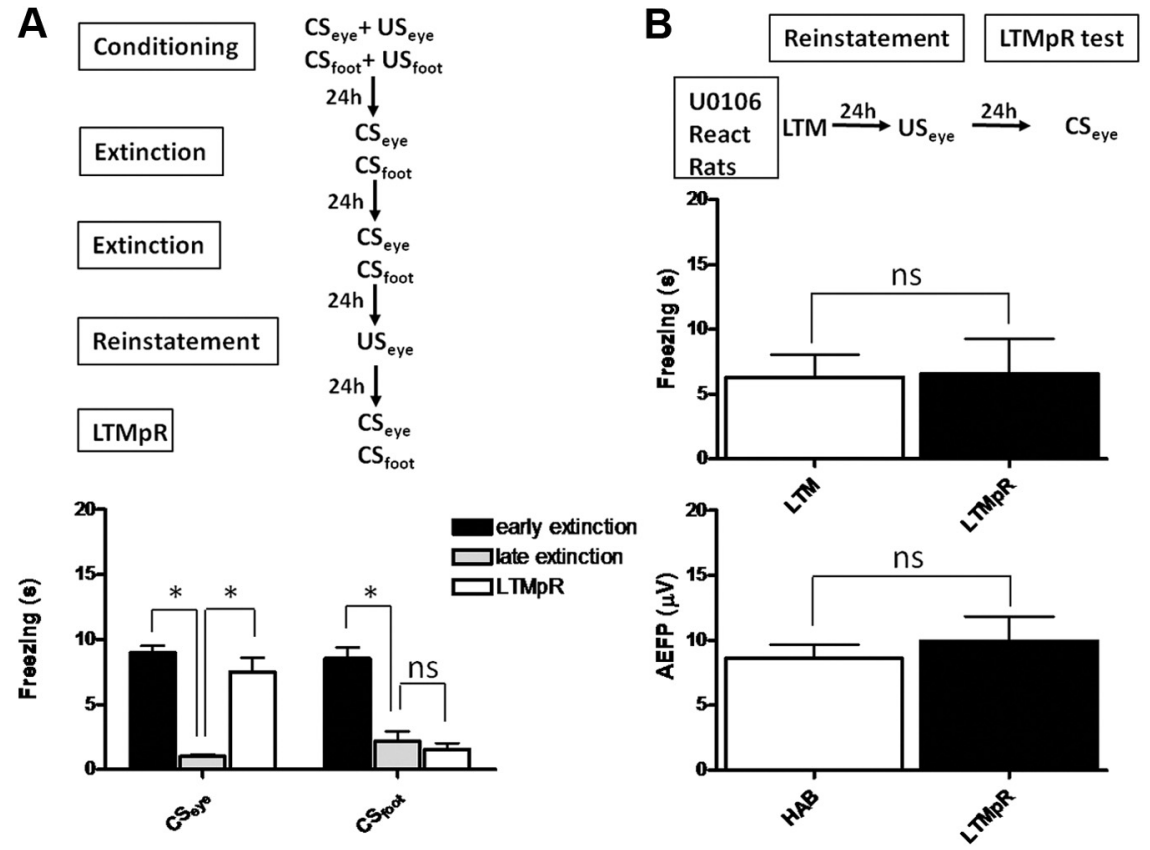

Figure 4. Once the US-triggered reconsolidation of fear memory and its CS neural representation is disrupted, a reinstatement procedure does not lead to their recovery. $\boldsymbol{A}$, Diagram showing the classic reinstatement procedure (top) used for testing the selectivity of reinstatement. Twenty-four hours after the animals $(n=7)$ were conditioned with the $\mathrm{CS}_{\text {eye }}-U \mathrm{~S}_{\text {eye }}$ and $\mathrm{CS}_{\text {foot }}-U \mathrm{~S}_{\text {foot }}$ protocol, they were submitted to 2 consecutive days of strong extinction with the presentation of $15 \mathrm{CS}_{\text {eye }}$ and $15 \mathrm{CS}_{\text {foot }}$ each day. The day after the last extinction session, one unsignaled $\mathrm{US}_{\text {eye }}$ was delivered to all the rats. Twenty-four hours later, all rats were tested for LTMPR memory. Freezing (mean \pm SEM) average at early extinction (first four (S presentations of the first extinction day, black bars), at late extinction (last four presentations of the second day of extinction, gray bars) and during LTMpR test (white bars) is represented in the bottom chart for $\mathrm{CS}_{\text {eye }}$ and $\mathrm{CS}_{\text {foot }}$. Only the freezing to the $\mathrm{CS}_{\text {eye }}$ was reinstated after the presentation of the unsignaled US ${ }_{\text {eye }}\left({ }^{*} p<0.05\right.$, Tukey post hoc test). $\boldsymbol{B}$, The UOR group was submitted to an unsignaled US ${ }_{\text {eye }} 24 \mathrm{~h}$ after LTM test. The day after, these rats were tested for LTMpR. Neither the freezing to the $\mathrm{CS}_{\text {eye }}$ nor the $\mathrm{CS}_{\text {eye }}-\mathrm{AEFP}$ amplitude during LTMpR test (black bars) was different from LTM test (white bars) ( ${ }^{*} p<0.05$, Student $t$ test). pared with habituation in all groups $\left(\mathrm{CS}_{\text {eye }}\right.$ test $[\mathrm{HAB}$ vs STM] main effect, $F_{(1,21)}=52.46, p<0.0001 ; \mathrm{CS}_{\text {foot }}$ test $[\mathrm{HAB}$ vs STM] main effect, $F_{(1,21)}=49.90, p<0.0001$ ) (Fig. $2 C, D$ ). Noticeably, all groups showed a similar level of potentiation (no group main ffect in $\mathrm{CS}_{\text {eye }}$ or $\mathrm{CS}_{\text {foot }}, F \mathrm{~s}<1$; no group $\times$ test interaction for $\mathrm{CS}_{\text {eye }}, F_{(2,21)}=1.84$, ns; or $\left.\mathrm{CS}_{\text {foot }}, F s<1\right)$ revealing that the reactivation with the $\mathrm{US}_{\text {eve }}$ did not induce a further potentiation in the LA in contrast to what has been previously observed after

Does depotentiation of LA CS-evoked responses occur in parallel with the blockade of US-evoked memory reconsolidation?

To measure the CS-evoked neural responses during LTM, $24 \mathrm{~h}$ after retrieval, fear responses for both CSs were recorded again in the same modified context used for STM. A selective impairment freezing to the CS associated with the US previously reactivated $\left(\mathrm{CS}_{\text {eve }}\right)$ was affected $\left(\mathrm{CS}_{\text {foot }}\right.$ no group main effect, $F \mathrm{~s}<1$, ns; $\mathrm{CS}_{\text {eye }}$ group main effect, $F_{(2,21)}=6.85, p<0.005$; Tukey pairment of long-term fear memory. Thus, there was a group effect for $\mathrm{CS}_{\text {foot }}(p<0.01)$ but not for $\mathrm{CS}_{\text {eye }}(p>0.3)$ (data not ). This result confirms, with an MAPK inhibitor, our previous results obtained with the protein synthesis inhibitor anisomycin (Debiec et al., 2010). Importantly, the memory loss went in parallel with a decrease in the amplitude of AEFP evoked by $\mathrm{CS}_{\text {eye }}$ (Fig. $3 \mathrm{C}$ ) (group $\times$ test [HAB vs LTM] interaction, $F_{(2,21)}=5.73, p<$ $0.05)$. This decrease required both the memory reactivation and the infusion of the MAPK inhibitor, as the amplitude of the AEFPs went back to baseline levels only for the U0R group (Tukey post hoc test $\mathrm{CS}_{\text {eye }}$ [HAB vs LTM], ns), while AEFPs for U0NR and VeR groups remained potentiated (Tukey post hoc test $\mathrm{CS}_{\text {eye }}[\mathrm{HAB}$ vs LTM], $p<0.05$ for each group). Furthermore, this depotentiation was selective to the CS associated to the reactivated US, as the AEFP to the $\mathrm{CS}_{\text {foot }}$ for the UOR group remained potentiated (Fig. $3 D$ ) (test [HAB vs LTM] main effect, $F_{(1,21)}=31.83, p<0.0001$; no group main effect, $F s<1$; no group $\times$ test interaction, $\left.F_{(2,21)}=1.2, \mathrm{~ns}\right)$. Together, these results show that US reactivation triggers a selective reconsolidation process affecting only the associated CS-evoked neurophysiological response in the LA.

Does the depotentiation of the CS representation in LA reflect a lasting disruption of the memory trace?

A key question is whether the loss of fear memory both at the behavioral and neurophysiological levels is due to a blockade in memory retrieval or to an impairment of the memory itself. A retrieval deficit is usually demonstrated by the reappear- 
ance of the memory in certain conditions, such as after a reinstatement procedure. The use of a reinstatement procedure in which a return of fear is triggered by the presentation of an unsignaled US has proven to be instrumental for demonstrating that memories formed during fear acquisition remain intact after fear extinction (Rescorla, 1966; Rescorla and Heth, 1975; Bouton, 1993). Therefore, we first tested, in a separate behavioral experiment, whether a single US presentation could reinstate the fear after it had been extinguished in a selective manner in our dualfear conditioning protocol. After conditioning with the $\mathrm{CS}_{\text {eve }}-$ $\mathrm{US}_{\text {eye }}$ and $\mathrm{CS}_{\text {foot }}-\mathrm{US}_{\text {foot }}$, all rats were submitted to two sessions of extinction, during which $\mathrm{CS}_{\text {eye }}$ and $\mathrm{CS}_{\text {foot }}$ were no longer followed by USs (Fig. 4A, top). Twenty-four hours after the last extinction session, a single $\mathrm{US}_{\text {eye }}$ was delivered, and fear memory to both CSs was tested the day after (LTMpR). Only the fear memory for the CS linked to the reinstated US $\left(\mathrm{CS}_{\text {eye }}\right)$ was recovered (Fig. $4 A$, bottom) $\left(\mathrm{CS}_{\text {eye }}\right.$ early vs late extinction vs LTMpR main effect, $F_{(2,12)}=29.57, p<0.0001$; Tukey post hoc, LTMpR vs late extinction, $p<0.001)$, leaving extinguished the fear responses to the other $\mathrm{CS}\left(\mathrm{CS}_{\text {foot }}\right)\left(\mathrm{CS}_{\text {foot }}\right.$ early vs late extinction vs LTMpR main effect, $F_{(2,12)}=27.93, p<0.0001$; Tukey post hoc; LTMpR vs late extinction, ns). A selective recovery was also observed when the fear was reinstated with unsignaled $\mathrm{US}_{\text {foot }}$ (return of fear to $\mathrm{CS}_{\text {foot }}$ and not to $\mathrm{CS}_{\text {eye }}$, data not shown), thus demonstrating that the effect was not due to particular characteristics of the US. Therefore, in our dual-fear conditioning protocol, reinstatement after extinction induced a selective rescue of CS fear memory, in agreement with cue-specific reinstatement previously reported (Rescorla and Heth, 1975; Bouton, 1993; Westbrook et al., 2002).

Using the reinstatement methodology, we then asked whether the fear memory impairment observed in animals for which the reconsolidation has been disrupted by the MAPK inhibitor could be alleviated (Fig. $4 B$ ) by US presentation. Twenty-four hours after LTM, animals from the UOR group for which fear memory to the $\mathrm{CS}_{\text {eye }}$ had diminished after the disruption of reconsolidation were submitted to a single US $_{\text {eye }}$, and long-term memory was again tested $24 \mathrm{~h}$ later (LTMpR). Neither fear memory (no LTM vs LTMpR effect, $F<1$ ) nor potentiation of AEFP associated with the $\mathrm{CS}_{\text {eye }}$ was recovered (no HAB vs LTMpR effect, $F_{(1,6)}=1.18$; ns) (Fig. $4 B$ ). This result suggests that the loss of fear memory and its neurophysiological correlate in the LA induced by the MAPK inhibitor after US reactivation did not reflect a memory retrieval blockade as it was not reinstated by US presentation. This is consistent with the absence of spontaneous recovery after 1 month in the same protocol using anisomycin as a pharmacological deconsolidation tool (Debiec et al., 2010). Our findings, therefore, demonstrate in parallel a fear memory loss and depotentiation of CS-evoked neurophysiological responses in the LA when USelicited reconsolidation processes are disrupted.

\section{Discussion}

The present results demonstrate that presentation of the aversive stimulus induces lability in the neural representation of its associated CS in the LA and makes the CS-evoked memory trace sensitive to disruption by an MAPK inhibitor. Neither the LA CS representation nor the behavioral fear memory recovered after a reinstatement procedure. This suggests that disrupting USelicited reconsolidation of fear memories results in a lasting alteration of the neural representation of the CS-US association in the LA. Once the association between the CS and US is created, the neural representations of the two stimuli may become a unique CS-US entity in the amygdala, thus opening the possibility for a selective erasure of CS-US associations by presenting either elements of the association. The question of whether the deconsolidation process involves a destabilization of plasticity only at CS-US convergence sites in LA remains to be elucidated.

In a similar paradigm, reactivation of fearful memory induced by the presentation of a tone CS induced a further potentiation of the LA CS-evoked field potentials (Doyère et al., 2007). No supplementary potentiation was observed in the $\mathrm{CS}_{\text {eye }}$-evoked potentials (the exact same pure tone) during STM in the present experiment. This may be due to differences in protocols (using two different USs here vs a single US $_{\text {foot }}$ in the previous study). Alternatively, this may be due to the fact that the US rather than the CS was used to trigger memory retrieval, and that additional potentiation is induced only at activated synapses during reactivation (here by the US and not the CS). In any case, the lack of retrieval-induced potentiation at CS-activated synapses together with the selective depotentiation of CS-evoked field potentials suggests that the two processes may be independent once initiated by the reactivating retrieval cue, possibly involving separate presynaptic and postsynaptic mechanisms. Further experiments will have to be specifically designed to address this question.

Several observations suggest that the disruption of LA reconsolidation processes produced a fear memory loss, rather than a retrieval deficit: the depotentiation of the CS-evoked field potential observed during LTM test; the fact that neither the fear memory, nor its associated potentiation in LA could be recovered after a reinstatement procedure; and our previous results showing no spontaneous recovery after a month in the same behavioral paradigm (Debiec et al., 2010). Therefore, in agreement with current theories, these results show that cued amygdala-dependent fear memories, when reactivated, undergo a reconsolidation process that is sensitive to selective alteration at the cellular level, at least in the case of the LA (Sara, 2000; Miller and Sweatt, 2006; Doyère et al., 2007; Hardt et al., 2009; Lee, 2009; Nader and Einarsson, 2010).

These new findings constitute an important step in our understanding how fear memories are organized and linked and suggest that presentation of the aversive event which initially triggered memory formation may allow access to and manipulation of associated traumatic memories. This approach could be used as an efficient therapeutic tool for the treatment of pathologies related to chronic pain, substance addiction, or posttraumatic stress disorders.

\section{References}

Bouton ME (1993) Context, time, and memory retrieval in the interference paradigms of Pavlovian learning. Psychol Bull 114:80-99.

Davis M (1997) Neurobiology of fear responses: the role of the amygdala. J Neuropsychiatry Clin Neurosci 9:382-402.

Debiec J, Doyère V, Nader K, Ledoux JE (2006) Directly reactivated, but not indirectly reactivated, memories undergo reconsolidation in the amygdala. Proc Natl Acad Sci U S A 103:3428-3433.

Debiec J, Díaz-Mataix L, Bush DE, Doyère V, Ledoux JE (2010) The amygdala encodes specific sensory features of an aversive reinforcer. Nat Neurosci 13:536-537.

Doyère V, Debiec J, Monfils MH, Schafe GE, LeDoux JE (2007) Synapsespecific reconsolidation of distinct fear memories in the lateral amygdala. Nat Neurosci 10:414-416.

Duvarci S, Nader K, LeDoux JE (2005) Activation of extracellular signalregulated kinase- mitogen-activated protein kinase cascade in the amygdala is required for memory reconsolidation of auditory fear conditioning. Eur J Neurosci 21:283-289.

Fanselow MS, LeDoux JE (1999) Why we think plasticity underlying Pav- 
lovian fear conditioning occurs in the basolateral amygdala. Neuron 23:229-232.

Fanselow MS, Poulos AM (2005) The neuroscience of mammalian associative learning. Annu Rev Psychol 56:207-234.

Hardt O, Wang SH, Nader K (2009) Storage or retrieval deficit: the yin and yang of amnesia. Learn Mem 16:224-230.

Konorski, J (1967) Integrative Activity of the Brain: An Interdisciplinary Approach. Chicago: University of Chicago.

LeDoux JE (2000) Emotion circuits in the brain. Annu Rev Neurosci 23:155-184.

Lee JL (2009) Reconsolidation: maintaining memory relevance. Trends Neurosci 32:413-420.

Maren S (2001) Neurobiology of Pavlovian fear conditioning. Annu Rev Neurosci 24:897-931.

Miller CA, Sweatt JD (2006) Amnesia or retrieval deficit? Implications of a molecular approach to the question of reconsolidation. Learn Mem 13:498-505.
Nader K, Einarsson EO (2010) Memory reconsolidation: an update. Ann N Y Acad Sci 1191:27-41.

Pavlov IP (1927) Conditional Reflexes. New York: Dover.

Rescorla RA (1966) Predictability and number of pairings in Pavlovian fear conditioning. Psychonomic Science 4:383-384.

Rescorla RA, Heth CD (1975) Reinstatement of fear to an extinguished conditioned stimulus. J Exp Psychol Anim Behav Process 1:88-96.

Rogan MT, Stäubli UV, LeDoux JE (1997) Fear conditioning induces associative long-term potentiation in the amygdala. Nature 390:604-607.

Sara SJ (2000) Retrieval and reconsolidation: toward a neurobiology of remembering. Learn Mem 7:73-84.

Schafe GE, Doyère V, LeDoux JE (2005) Tracking the fear engram: the lateral amygdala is an essential locus of fear memory storage. J Neurosci $25: 10010-10014$.

Westbrook RF, Iordanova M, McNally G, Richardson R, Harris JA (2002) Reinstatement of fear to an extinguished conditioned stimulus: two roles for context. J Exp Psychol Anim Behav Process 28:97-110. 